Original Paper http://ajol.info/index.php/ijbcs http://indexmedicus.afro.who.int

\title{
Caractérisation des sols de Guillé Koira et Lassourou dans les communes rurales d'Imanan et Tondikandia au Niger pour une meilleure culture de riz autour des mares.
}

\author{
Amir SIDO YACOUBA ${ }^{1 *}$, Guero YADJI ${ }^{2}$, Mella MAMANE TCHICAMA ${ }^{3}$ et \\ Nourou ABDOURAHAMANE ISSA ${ }^{4}$
}

\footnotetext{
${ }^{I}$ Institut National de la Recherche Agronomique du Niger (INRAN), BP 429, Niamey, Niger.

${ }^{2}$ Faculté d'Agronomie de l'Université Abdou Moumouni de Niamey, BP 10960, Niamey, Niger.

${ }^{3}$ Faculté des Sciences de l'Université Abdou Moumouni de Niamey, Niger.

${ }^{4}$ Etudiant à la Faculté d'Agronomie de l'Université Abdou Moumouni de Niamey, Niger.

*Auteur correspondant ; E-mail: sidoamir@yahoo.fr; Tel: +22796961175
}

\section{RESUME}

Dans les communes rurales de Tondikandia et d'Imanan situées dans le département de Filingué luimême rattaché à la région de Tillaberi, il existe des mares permanentes dont les eaux sont sous exploitées du fait de la méconnaissance des sols pour une bonne production de riz autour de ces mares. Notre travail a été réalisé au cours de la saison d'hivernage 2015 avec comme objectif de caractériser les sols de sites de Guillé Koira et de Lassourou situés dans ces deux communes rurales pour mieux pratiquer la culture de riz. Pour se faire, des prélèvements des échantillons du sol ont été effectués au niveau des fosses pédologiques préalablement décrites au niveau des sites concernés. Ces échantillons ont été analysés. Des principaux résultats obtenus, on retiendra que les sols utilisés ont des caractéristiques physico-chimiques différentes. A Guillé koira, le pH est acide sur les premiers horizons. Cette acidité $(5,05)$ dépasse 5,5 valeur en dessous de laquelle la présence de l'aluminium échangeable présente des risques de toxicités aluminiques. A Lassourou, L'analyse chimique a révélé un $\mathrm{pH}$ basique dans les horizons de surface. Le $\mathrm{pH}(9,2)$ et la conductivité électrique ( $\mathrm{CE}=75)$ d'une part et la prédominance de la texture grossière en surface d'autre part sont des facteurs préjudiciables à la riziculture. En conclusion on peut retenir que la riziculture est possible à Guillé Koira et Lassourou. Cependant à Lassourou, des amendements notamment l'apport d'eau régulier en période de poche de sécheresse, l'utilisation rationnelle de gypse, de fumure organique et des variétés tolérantes à la salinité de sol permettront d'obtenir une bonne production du riz en saison des pluies.

(C) 2018 International Formulae Group. All rights reserved.

Mots clés: Niger, caractérisation, sols, riziculture autour des mares, $\mathrm{pH}, \mathrm{CE}$.

\section{Soils characterization of Guillé Koira and Lassourou in the rural communes of Imanan and Tondikandia in Niger for better rice production around the pools}




\begin{abstract}
In the rural communes of Tondikandia and Imanan located in the department of Filingue itself attached to the Tillaberi region, there exist permanent ponds whose water is under exploited due to the lack of knowledge of the soils for a good rice production around these ponds. Our work was realized during the wintering season of 2015 with a goal to characterize the soils of Guillé Koira and Lassourou located in these two rural communes to better practice the production of rice. To start with, selections of soils samples were carried out on the pedological pits described beforehand on the level of the concerned sites. These samples were analyzed. From the principal results obtained, it will be retained that the used soils have different physicochemical characteristics. In Guille koira, the $\mathrm{pH}$ is acid on the first horizons. This acidity (5.05) exceeds 5.5 value below which the presence of exchangeable aluminium presents the aluminic toxicities risks. In Lassourou, the chemical analysis revealed a basic $\mathrm{pH}$ in the surface horizons. The $\mathrm{pH}(9.2)$ and electric conductivity $(\mathrm{EC}=75)$ on the one hand, the predominance of coarse texture on the surface on the other hand are prejudicial factors to the rice production. In conclusion we can retain that rice growing is possible in Guillé Koira and Lassourou. However, in Lassourou, some amendments in particular the bringing of regular supply of water during the period of dry pockets, the rational use of gypsum, organic fertilizer and the tolerant varieties to the soil salinity will allow to obtain a good production of rice in rainy season.
\end{abstract}

(C) 2018 International Formulae Group. All rights reserved.

Keywords: Niger, characterization, soils, rice growing around the pools, $\mathrm{pH}, \mathrm{EC}$.

\section{INTRODUCTION}

$\mathrm{Au}$ Niger, la culture du riz est pratiquée essentiellement dans la vallée du fleuve Niger, notamment dans les régions de Tillabéri, Niamey et Dosso. La production céréalière nationale du riz est estimée à 132.030 tonnes (FAO, 2011) tandis que sa consommation entre de plus en plus dans les habitudes alimentaires des populations tant en milieu rural qu'en milieu urbain avec un record à Niamey estimée à 41,27 $\mathrm{Kg} /$ habitant/an (INS, 2011). C'est en ce sens que la riziculture traditionnelle autour des mares et d'autres points d'eau commence à se développer particulièrement dans les départements de Ouallam et de Fillingué (région de Tillabéri).

La riziculture de mare est une culture pluviale pratiquée dans des superficies modestes avec de rendements moyens de l'ordre de 0,7 t/ha (PAFRIZ, 2005). Cependant, depuis 2010, l'expérience des jeunes de Ouallam (région de Tillaberi) appuyés par l'ONG AGDL (Action des Groupements pour le Développement Local.) a montré sur le site de Fari Beri (Commune rurale de Simiri) que ces rendements peuvent atteindre 1,5 t/ha (AGDL, 2010). Cette riziculture est conduite sans aucune connaissance des caractéristiques des sols des sites où elle est pratiquée.

L'objectif de l'étude est de caractériser des sols des sites de Guillé Koira et de Lassourou dans les communes rurales d'Imanan et Tondikandia (Département de Filingué) pour mieux pratiquer la culture de riz autour des mares.

\section{MATERIEL ET METHODES Matériel}

Le matériel se compose de :

- $\quad$ outils pour creuser les profils et prélever des échantillons: bêches, pelles, pioches, pics et soudes (les tarières hélicoïdales) ;

- matériels d'étude et d'observation: piochon de pédologue (rafraîchir et protéger les échantillons), double mètre pour mesurer la profondeur et l'épaisseur, flacon d'eau ; flacon d'acide chlorhydrique dilué (pour apprécier la présence et la charge en calcaire) ;

- tarière pour les prélèvements des échantillons du sol ;

- code de couleur MUNSELL, appareil photographique numérique ;

- $\quad$ fiches de description; 
GPS pour relever les coordonnées de délimitation du site et position des profils.

\section{Méthodes \\ Prélèvement des échantillons}

Les échantillons de sols sont prélèvés sur les deux sites (Guillé Koira et Lassourou). Chaque site est subdivisé en deux parties : la partie cultivée en saison des pluies et celle cultivée en saison sèche. Sur les deux parties de chaque site, des prélèvements de sols à la tarière et dans les fosses pédologiques ont été effectués. Ainsi au total quinze (15) échantillons ont été collectés.

\section{Analyses de laboratoire}

Les analyses des échantillons de sols prélevés sur chaque site ont été effectuées dans le laboratoire du département de Science du sol de la Faculté d'agronomie de l'Université Abdou Moumouni de Niamey. Elles ont porté sur la granulométrie, les analyses physicochimiques et la conductivité électrique.

Ces échantillons prélevés sur les sites ont été séchés à la température ambiante du laboratoire et tamisés à $2 \mathrm{~mm}$. Les analyses ont été effectuées sur la terre fine. Les classes texturales sont déterminées selon la nomenclature de l'U.S. Department of Agriculture et les analyses chimiques sont interprétées en référence au guide d'interprétation des analyses de sol adopté par la FAO (1992).

Les analyses physico-chimiques ont permis de déterminer: la granulométrie, le pH-eau, le carbone organique total (C), l'Azote total $(\mathrm{N})$, le Phosphore total et assimilable $(\mathrm{P})$, le potassium total $(\mathrm{K})$, la conductivité électrique (CE), la capacité d'échange cationique (CEC) et les bases échangeables $(\mathrm{Ca}++, \mathrm{Mg}+, \mathrm{Na}$ et $\mathrm{K}+)$.

\section{Analyse granulométrique}

La granulométrie a été déterminée par la méthode de la pipette Robinson. Son but est de déterminer la composition du sol en classes de tailles des particules. Cette méthode consiste à intercepter les fronts de sédimentation des particules fines constituées des argiles et des limons, suivis du tamisage mécanique pour les éléments grossiers (sable).

\section{pHeau}

La mesure du pHeau s'effectue à l'aide d'une électrode de verre combinée trempant dans une suspension de sol dans un rapport sol/eau= $1 / 2.5$ avec un $\mathrm{pH}$-mètre après 2 heures de contact. Ainsi on détermine le pHeau.

\section{Conductivité électrique (CE)}

La conductivité électrique est mesurée à l'aide d'un conductivimètre sur une suspension de sol dans un rapport (sol/eau) : $1 / 5$ après 2 heures de contact, et la lecture est effectuée avec un conductivimètre. Les résultats sont donnés en $\mu \mathrm{s} / \mathrm{cm}$.

\section{Bases échangeables (Ca, $\mathrm{Mg}, \mathrm{Na}, \mathrm{K}$ )}

Un échantillon de $10 \mathrm{~g}$ de terre fine est transvasé dans un percolateur préalablement muni d'un filtre. Il est d'abord saturé avec une solution d'acétate d'ammonium $1 \mathrm{~N}$ pendant 18 heures au moins, pour déplacer les ions $\mathrm{Ca}^{2+}, \mathrm{Mg}^{2+}, \mathrm{Na}^{+}, \mathrm{K}^{+}$qui se trouvent sur le complexe absorbant et qui seront remplacé à leur tour par les ions $\mathrm{NH}_{4}{ }^{+}$ absorbant. Ensuite, on place sous le percolateur, une fiole de $100 \mathrm{ml}$ et on ouvre son robinet et le filtrat contenant les bases échangeables tombe par goutte dans la fiole jusqu'au trait de jauge de $100 \mathrm{ml}$.

\section{CEC}

L'extraction des bases, $100 \mathrm{ml}$ d'alcool à $95{ }^{\circ} \mathrm{C}$ sont versés dans le percolateur pour entraîner l'excès de l'acétate d'ammonium qui se trouve dans la porosité. On ajoute du $\mathrm{KCl} 1 \mathrm{~N}$ pour déplacer les ions $\mathrm{NH}_{4}{ }^{+}$qui se trouvent sur le complexe absorbant. La solution contenant les ions ammonium déplacés est recueillie dans une fiole de $250 \mathrm{ml}$. Le dosage des ions ammonium va permettre de déterminer la capacité d'échange cationique. 


\section{Phosphore total}

A un échantillon de $5 \mathrm{~g}$ de terre fine, on ajoute $10 \mathrm{ml}$ d'acide nitrique puis on le place sur une plaque chauffante pendant 5 heures et suivi de refroidissement. On ajoute $100 \mathrm{ml}$ d'acide sulfurique $1 \mathrm{~N}$ dans le bécher contenant la prise par fraction, et le surnageant est filtré sur papier filtre Watman.

A $5 \mathrm{ml}$ d'extrait du phosphore, on ajoute $10 \mathrm{ml}$ de molybdate d'ammonium, et compléter avec $33 \mathrm{ml}$ d'eau distillée pour faire $50 \mathrm{ml}$ de solution. On laisse reposer pendant 30 minutes pour obtenir la couleur jaune et on mesure l'absorbance au spectrophotocolorimètre.

\section{Phosphore assimilable}

On ajoute à un échantillon de $5 \mathrm{~g}$ de terre fine, $35 \mathrm{ml}$ une solution d'un mélange de difluorure d'ammonium et acide chlorhydrique. On agite pendant une minute et on procède à la filtration sur papier filtre. 5 $\mathrm{ml}$ d'extrait sont mélangés avec $10 \mathrm{ml}$ de molybdate d'ammonium, et $2 \mathrm{ml}$ de chlorure de cyanure (comme indicateur), plus $33 \mathrm{ml}$ d'eau distillée pour compléter à $50 \mathrm{ml}$. On laisse reposer pendant 30 minutes pour la révélation de la couleur bleue et ensuite on fait la lecture de la couleur avec un spectrophotocolorimètre.

\section{Carbone organique}

Le carbone organique est mesuré par la méthode décrite par Walkey et Black, basée sur l'oxydation en milieu acide du carbone organique par le dichromate de potassium. Les teneurs en carbone organique sont mesurées par titration. La teneur en matière organique s'obtient en multipliant le carbone organique par le facteur Sprengel (2 pour les sols remaniés et 1,724 pour les sols sous végétation naturelle).

\section{Analyse des données}

Les données collectées ont été analysées à l'aide du logiciel Gen Stat (treizième édition).

\section{RESULTATS}

Caractérisation des sols de Guillé Koira

Caractérisation morphologique du Profil No 1 (PGK 1)

La Figure1 ci-dessous illustre le profil PGK 1 (A) et l'état de surface du sol (B) avant l'ouverture de la fosse du site de Guillé koira.

Cette partie du site représente le lit majeur de la mare endoréique et est couvert par une végétation herbacée et ligneuse: Faidherbia albida, Penisetum amerrinum et culture du riz.

Les caractéristiques morphologiques du profil No 1 (PGK1) ouvert sur cette partie sont pour de $0-10 \mathrm{~cm}$ : horizon de couleur jaune rougeâtre $(7,5 \mathrm{YR} 6 / 6)$ à sec, texture sableuse légèrement limoneuse, structure massive, consistance fragile, poreux horizon peu organique, présence de quelques fines racines horizontales, limite progressive et linéaire;

De 10-30 cm: horizon de couleur brun foncé $(7,5 \mathrm{YR} 5 / 6)$ à l'état frais, texture sablo-limoneuse, structure massive, consistance peu dure, peu poreux, horizon très peu organique, présence de quelques rares racines très fines ;

De 30-90 cm plus: horizon de couleur rouge jaunâtre $(5 \mathrm{YR} 5 / 8)$ à l'état humide, texture sablo-limoneuse, structure massive, consistance fragile à l'état humide, quelques alvéoles et quelques rares galeries de termites.

La prédominance de la couleur rougejaunâtre est due à la présence des matériaux ferrugineux dans l'environnement de la mare.

Caractérisation physico-chimique du profil No 1 (PGK 1)

Les résultats de l'analyse physico-chimiques des trois horizons du profil No 1 (PGK 1) de Guillé Koira sont présentés dans le Tableau 1. Ce tableau montre que, les valeurs obtenues dans l'analyse granulométrique présentent une dominance du sable fins et du sable moyens sur toutes les autres textures, 
variant respectivement de 38,61 à 43,47 et de 34,2 à 42,46. Ces sols ont une texture sableuse (sable > 70\%) pour tous les horizons; ce qui est en accord avec les caractéristiques morphologiques. Les valeurs du $\mathrm{pH}$ sont acides et varient de 4,16 à 4,4. L'horizon (10-30) présente la valeur la plus élevée (4,4). Les teneurs en carbone organique et en azote du profil PGK 1 sont très faibles, et varient respectivement de 0,18 en surface et de 0,01 en profondeur. Le carbone organique de ce sol étant inférieure à $3 \%$, et le rapport $\mathrm{C} / \mathrm{N}$ très bas $(11,34$ à 11,62 ), alors ce sol est pauvre en matière organique. Les valeurs de la capacité d'échange cationique (CEC) obtenues sont constantes $(7,5)$, ce qui est en accord avec la faible teneur en argile.

\section{Caractérisation morphologique du profil No 2 (PGK 2)}

La Figure 2 illustre le profil PGK 2 (A) et l'état de surface du sol (B) avant l'ouverture de la fosse du site de Guillé koira.

Cette partie du site se situe dans le lit mineur de la mare endoréique, et est occupée par une végétation herbacée et ligneuse : Faidherbia albida et culture du riz.

Les caractéristiques morphologiques du profil No 2 (PGK 2) ouvert sur cette partie sont :

0-20 cm : horizon de couleur brun foncé (7,5YR 5/8) à l'état frais, texture sablolimoneuse, structure massive, consistance peu dure, poreux avec une macroporosité tubulaire et galeries des termites, horizon peu organique, présence de quelques rares racines très fines, limite nette et rectiligne ;

20-65 cm plus : horizon de couleur rouge jaunâtre (5YR 4/6) à l'état humide, texture sablo-limoneuse, structure massive, consistance fragile, rares pores tubulaires.

30-60 cm: horizon de couleur brun foncé jaunâtre (10YR 3/4) à l'état humide, texture argilo-sableuse, structure massive, présences de nombreuses concrétions ferrugineuses vers le bas, présence de quelques grosses et moyennes racines horizontales, et de nombreuses traces de racines mortes; $69 \mathrm{~cm}$ plus : nappe phréatique.

\section{Caractéristiques physico-chimiques du} profil No 2 (PGK 2)

Le Tableau 2 présente les résultats de l'analyse physico-chimiques des deux horizons du profil No 2 (PGK 2) de Guillé Koira. Le résultat de ce tableau montre que les valeurs obtenues dans l'analyse granulométrique présentent aussi une dominance du sable fins et du sable moyens sur toutes les autres textures, variant respectivement de 37,85 à 45,91 et de 30,63 à 40,96. La texture est sablo-limoneuse pour tous les horizons. Les valeurs du $\mathrm{pH}$ varient de 4,76 en surface et de 5 en profondeur, c'est donc un sol acide. Le carbone organique et l'azote du profil PGK 2 sont très faibles, et varient respectivement de 0,16 à 0,28 et de 0,01 à 0,02 . La teneur en carbone organique est inférieure à $3 \%$, et le rapport $\mathrm{C} / \mathrm{N}$ est aussi faible $(11,39$ à 11,69), ce qui indique que ce sol est pauvre en matière organique. Les valeurs de la capacité d'échange cationique (CEC) obtenues sont constante (10), ce qui confirme aussi la faible teneur en argile de ce sol obtenu lors de l'analyse granulométrique.

\section{Caractéristiques chimiques $d u$ dispositif après la récolte}

Les résultats de l'analyse chimique de quatre parcelles du dispositif de Guillé Koira prélevés à la tarière de $20 \mathrm{~cm}$ après la récolte, sont présentés dans le Tableau 3. Ce tableau montre que les valeurs du $\mathrm{pH}$ sont acides et varient de 6,1 à 5,0. La valeur du $\mathrm{pH}$ la plus acide $(5,05)$ est observée au niveau de R2V7. Les teneurs en carbone organique et en azote de ces parcelles sont très faibles, respectivement de 0,32 à 0,76 et de 0,03 à 0,07 . Le carbone organique de ce sol étant inférieure à $3 \%$, et le rapport $\mathrm{C} / \mathrm{N}$ très bas $(0,56$ à 0,72$)$ donc ce sol est pauvre en matière organique. Les valeurs de la capacité 
d'échange cationique (CEC) dans la zone de concentration des racines varient 7,5 à 10 . Le Tableau 3 montre les caractéristiques chimiques de quatre casiers du dispositif de Guillé koira après la récolte.

\section{Caractérisation des sols du site de Lassourou}

La Figure 3 illustre le profil PLAS 1 (A) et l'état de surface du sol (B) avant l'ouverture de la fosse du site de Lassourou. Cette partie du site représente le lit majeur de la mare de Lassourou. Elle est couverte par une végétation herbacée et ligneuse : Faidherbia albida, Hyphaena thebaica, Balamites aegyptica, cyperus rotendus. C'est la partie inexploitée de la mare qui est colonisée par une espèce envahissante Typha australis.

Les caractéristiques morphologiques du profil No 1 (PLAS 1) sont :

0-30 cm : horizon de couleur brun foncé jaunâtre (10YR 4/4) à l'état frais, texture sableuse, structure massive, consistance fragile, horizon peu organique, présence de nombreuse racines moyens et quelques fines racines, limite progressive, présence des taches rouilles ;

30-60 cm : horizon de couleur brun foncé jaunâtre (10YR 3/4) à l'état humide, texture argilo-sableuse, structure massive, présences de nombreuses concrétions ferrugineuses vers le bas, présence de quelques grosses et moyennes racines horizontales, et de nombreuses traces de racines mortes; 69 cm plus: nappe phréatique.

\section{Caractéristiques physico-chimiques du profil No 1 (PLAS 1)}

Le Tableau 4 présente les résultats de l'analyse physico-chimiques du profil No 1 (PLAS 1) de Lassourou. Ce tableau montre que les valeurs granulométriques obtenues présentent aussi une dominance du sable fins et du sable moyens sur toutes les autres textures, variant respectivement de 32,38 à 36,13 et de 35,05 à 39,46 . La texture est sableuse pour tous les deux horizons. Les valeurs du $\mathrm{pH}$ sont basiques et augmentent avec la profondeur et devient de plus en plus alcalins $(8,25$ à 9,4$)$; c'est donc un sol salin. Le carbone organique et l'azote du profil PLAS 1 sont très faibles et varient respectivement de 0,24 à 0,31 et de 0,02 à 0,03 . La teneur en carbone organique est inférieure à $3 \%$, et le rapport $\mathrm{C} / \mathrm{N}$ est aussi faible $(11,44$ à 11,59$)$, ce qui indique que ce sol est pauvre en matière organique. Les valeurs de la conductivité électronique (CE) obtenues varient de 75 à 122.

\section{Caractéristiques chimiques du dispositif après la récolte}

Les résultats de l'analyse physicochimiques de quatre casiers du dispositif de Lassourou après la récolte sont présentés dans le Tableau 5. De ce tableau, il ressort que :

Les valeurs $\mathrm{du} \mathrm{pH}$ sont basiques et varient de 7,45 à 9,8 en fonction des casiers. La valeur du $\mathrm{pH}$ la plus basique $(9,8)$ est observée au niveau de R2V8 ;

les teneurs en carbone organique de ces casiers sont très faibles, et varient de 0,1 à 0,13 et celles de l'azote est constante 0,01 . Le carbone organique de ce sol étant inférieure à $3 \%$, et le rapport $\mathrm{C} / \mathrm{N}$ très bas $(12,15$ à 12,59$)$ donc ce sol est pauvre en matière organique ;

les valeurs de la conductivité électronique (CE) obtenues varient de 30 à 60 ; ce qui confirme les valeurs de $\mathrm{pH}$ obtenues ;

- les valeurs obtenues dans l'analyse granulométrique présentent une dominance du sable fins et du sable moyens sur toutes les autres textures, variant respectivement de 36,61 à 41,55 et de 38,45 à 44,02. La texture est sablo-limoneuse. 

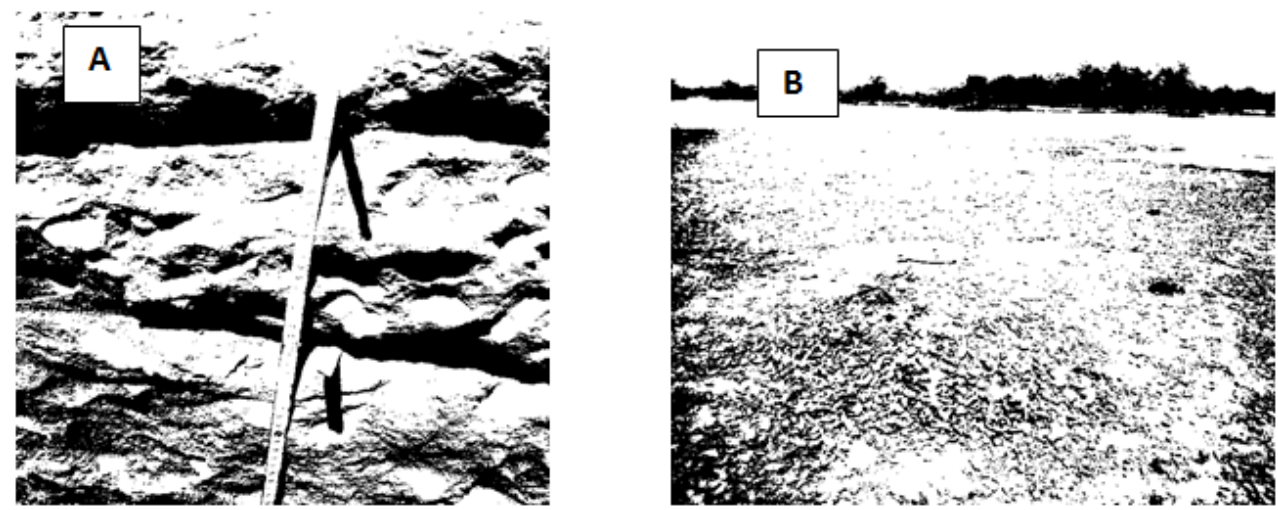

Figure 1: Les horizons de la fosse, Guillé Koira Profil No 1 (PGK 1) (A) et état de surface du sol (B).

Tableau 1: Caractéristiques physico-chimiques des trois horizons du profil No 1 (PGK 1) de Guillé Koira.

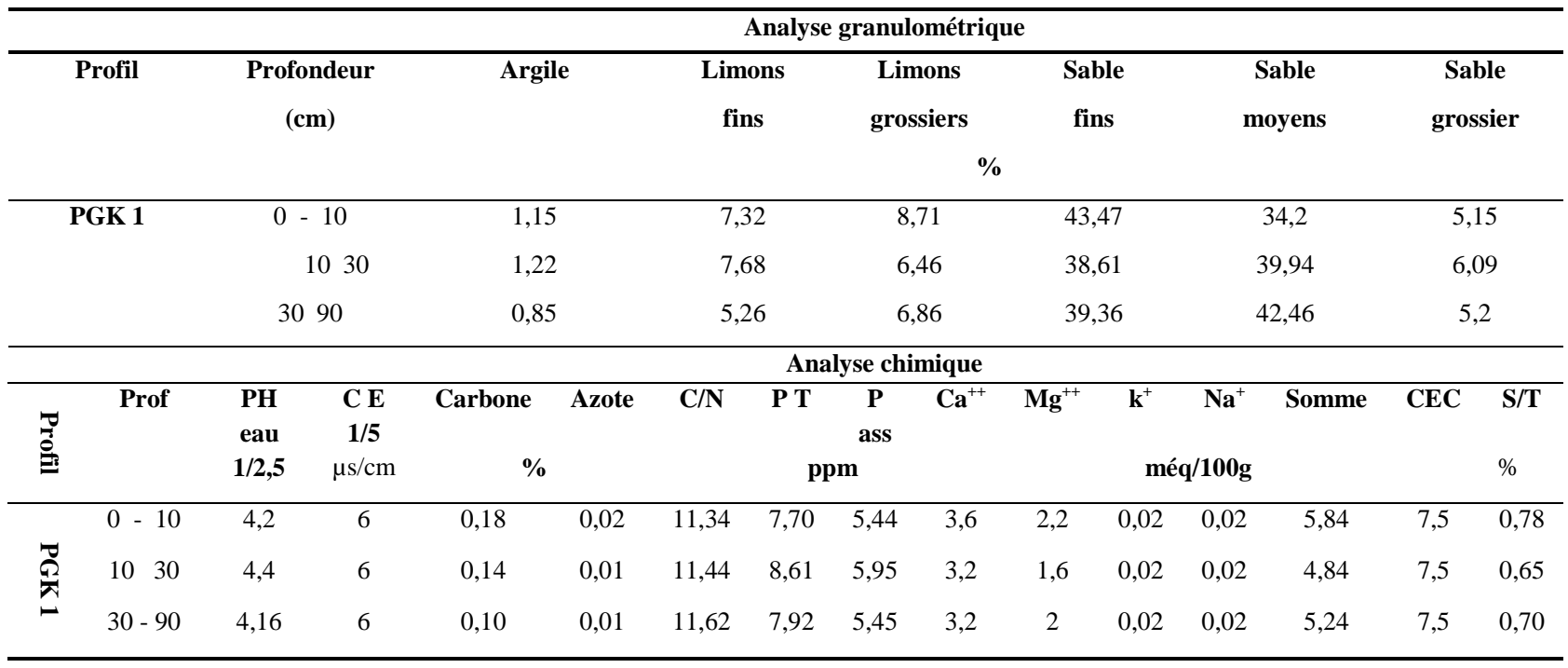
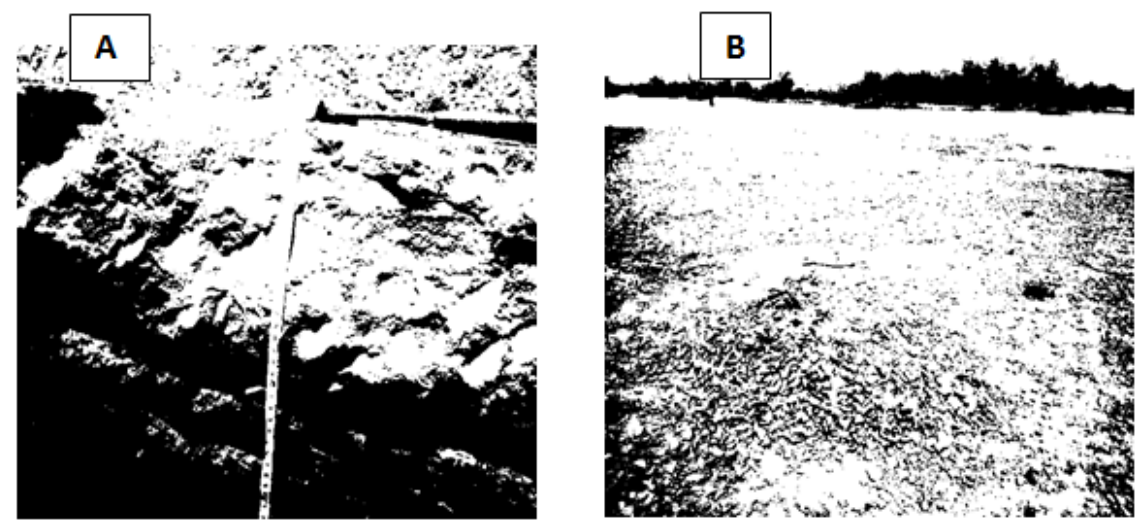

Figure 2: Les horizons de la fosse, Guillé Koira Profil No 2 (PGK 2) (A) et état de surface du sol (B). 
Tableau 2: Caractéristiques physico-chimiques des deux horizons du profil No 2 (PGK 2) de Guillé Koira.

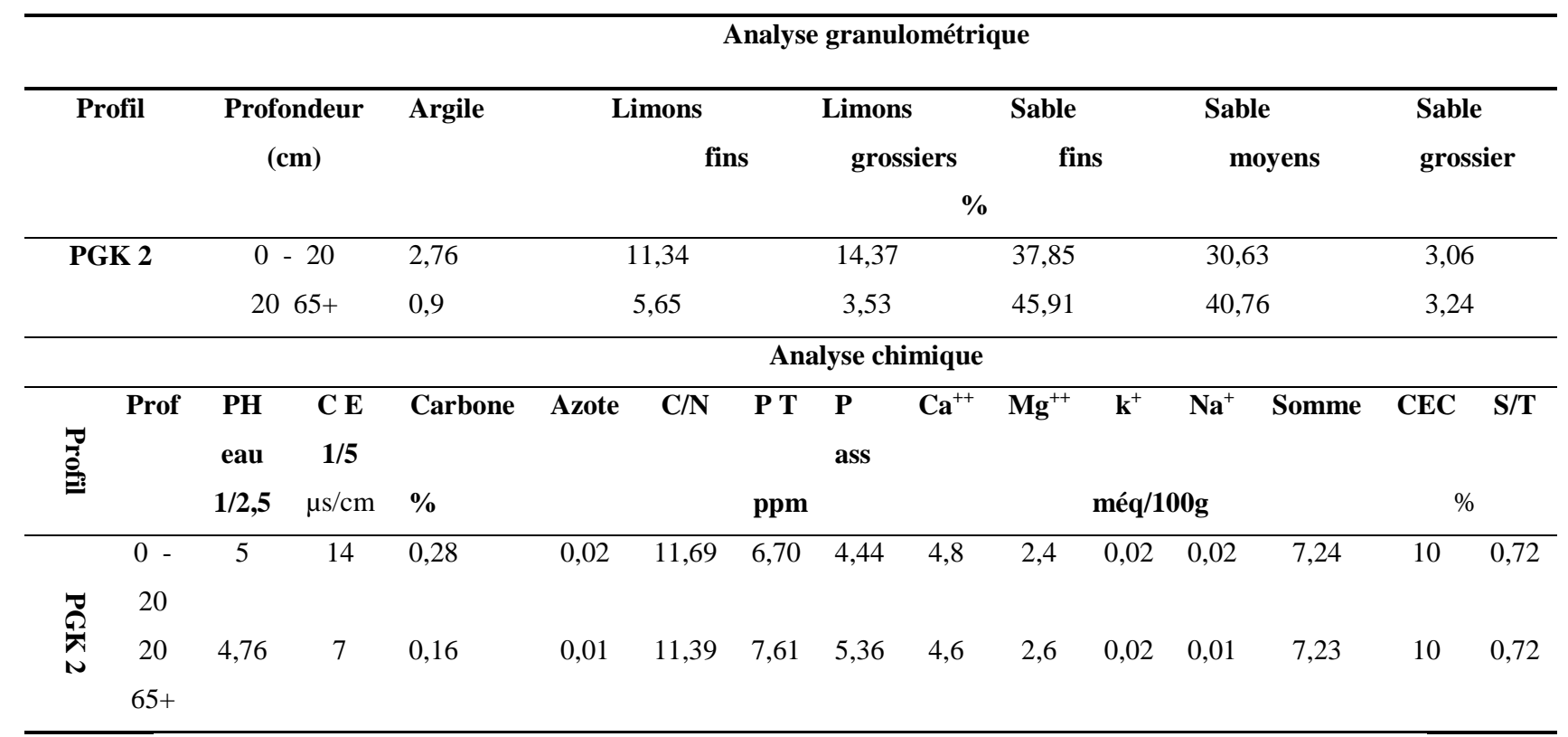

Tableau 3: Caractéristiques chimiques de quatre casiers du dispositif de Guillé Koira après la récolte.

\begin{tabular}{|c|c|c|c|c|c|c|c|c|c|c|c|c|c|c|c|}
\hline \multirow[b]{2}{*}{ Profil } & \multirow[b]{2}{*}{ Prof } & \multicolumn{14}{|c|}{ Analyse chimique } \\
\hline & & \multirow{3}{*}{$\begin{array}{c}\text { PH } \\
\text { eau } \\
1 / 2,5\end{array}$} & \multirow{3}{*}{$\begin{array}{c}\mathbf{C} \mathbf{E} \\
\mathbf{1 / 5} \\
\mu \mathrm{s} / \mathrm{cm}\end{array}$} & \multirow[t]{2}{*}{ Carbone } & \multirow[t]{3}{*}{ Azote } & \multirow[t]{2}{*}{$\mathbf{C} / \mathbf{N}$} & \multirow[t]{2}{*}{$\bar{P} \mathbf{T}$} & \multirow{2}{*}{$\begin{array}{c}\mathbf{P} \\
\text { ass }\end{array}$} & \multirow[t]{2}{*}{$\mathrm{Ca}^{++}$} & \multirow[t]{2}{*}{$\overline{\mathbf{M g}^{++}}$} & \multirow[t]{2}{*}{$\mathbf{k}^{+}$} & \multirow[t]{2}{*}{$\mathrm{Na}^{+}$} & \multirow[t]{2}{*}{ Somme } & \multirow[t]{3}{*}{ CEC } & \multirow[t]{2}{*}{$\mathrm{S} / \mathrm{T}$} \\
\hline & & & & & & & & & & & & & & & \\
\hline & & & & $\%$ & & & \multicolumn{3}{|c|}{ ppm } & \multicolumn{4}{|c|}{ méq/100g } & & $\%$ \\
\hline R2V5 & $0-$ & 6,1 & 15 & 0,76 & 0,07 & 11,18 & 9,33 & 6,19 & 2,6 & 1,6 & 0,02 & 0,01 & 4,23 & 7,5 & 0,56 \\
\hline & 20 & & & & & & & & & & & & & & \\
\hline R1V9 & $0-$ & 5,6 & 9 & 0,64 & 0,06 & 11,21 & 8,81 & 6,07 & 3 & 1,8 & 0,03 & 0,01 & 4,84 & 7,5 & 0,65 \\
\hline & 20 & & & & & & & & & & & & & & \\
\hline R1V4 & $0-$ & 5,55 & 8 & 0,52 & 0,05 & 11,26 & 7,84 & 6,30 & 4,8 & 1,6 & 0,03 & 0,02 & 6,45 & 8,25 & 0,78 \\
\hline & 20 & & & & & & & & & & & & & & \\
\hline R2V7 & $0-$ & 5,05 & 13 & 0,32 & 0,03 & 11,43 & 9,57 & 7,03 & 4,6 & 2,6 & 0,02 & 0,02 & 7,24 & 10 & 0,72 \\
\hline & 20 & & & & & & & & & & & & & & \\
\hline
\end{tabular}



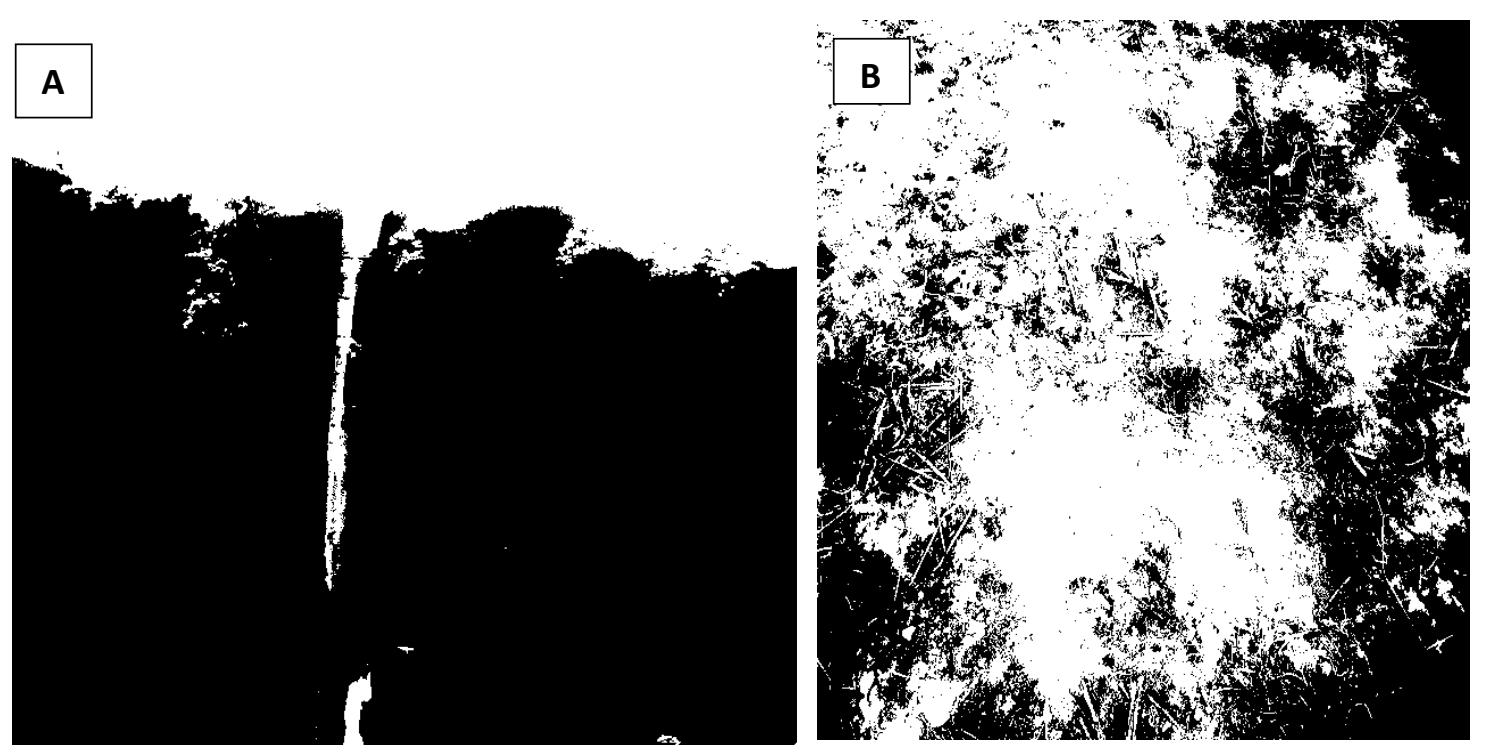

Figure 3: Les horizons de la fosse, Lassourou profil No 1 (PLAS 1) (A) et état de surface du sol (B).

Tableau 4: Caractéristiques physico-chimiques des deux horizons du profil No 1 (PLAS 1) de Lassourou .

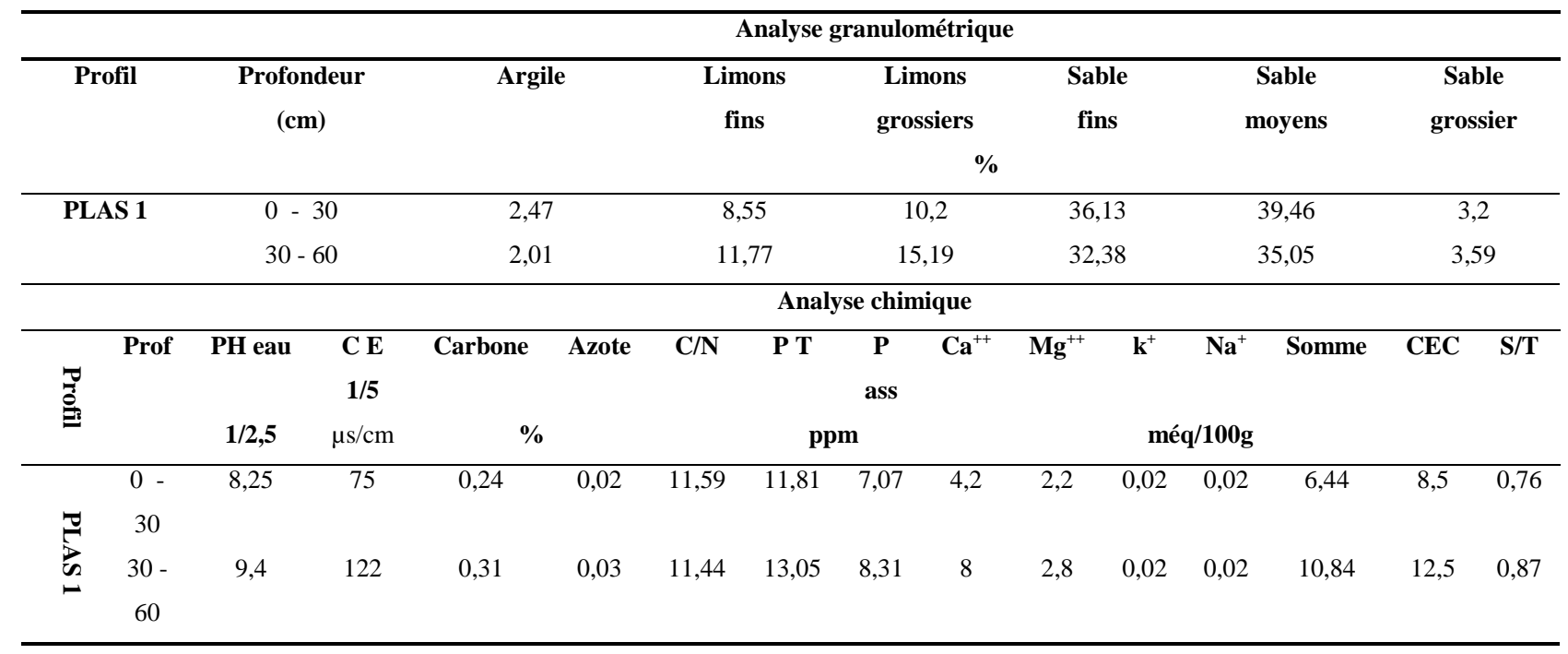


Tableau 5 : Caractéristiques chimiques de quatre casiers du dispositif de Lassourou après la récolte.

\begin{tabular}{|c|c|c|c|c|c|c|c|c|c|c|c|c|c|c|c|}
\hline \multirow[b]{2}{*}{ Profil } & \multirow{4}{*}{ Prof } & \multicolumn{14}{|c|}{ Analyse chimique } \\
\hline & & \multirow{3}{*}{$\begin{array}{c}\text { PH } \\
\text { eau } \\
1 / 2,5\end{array}$} & \multirow{3}{*}{$\begin{array}{c}\mathbf{C E} \\
\mathbf{1 / 5} \\
\mu \mathrm{s} / \mathrm{cm}\end{array}$} & \multirow[t]{2}{*}{ Carbone } & \multirow[t]{2}{*}{ Azote } & \multirow[t]{2}{*}{$\mathbf{C} / \mathbf{N}$} & \multirow[t]{2}{*}{$\mathbf{P} \mathbf{T}$} & \multirow{2}{*}{$\begin{array}{c}\mathbf{P} \\
\text { ass }\end{array}$} & \multirow[t]{2}{*}{$\mathrm{Ca}^{++}$} & \multirow[t]{2}{*}{$\mathbf{M g}^{++}$} & \multirow[t]{2}{*}{$\mathbf{k}^{+}$} & \multirow[t]{2}{*}{$\mathrm{Na}^{+}$} & \multirow[t]{3}{*}{ Somme } & \multirow[t]{3}{*}{ CEC } & \multirow[t]{2}{*}{$\mathbf{S} / \mathbf{T}$} \\
\hline & & & & & & & & & & & & & & & \\
\hline & & & & \multicolumn{2}{|c|}{$\%$} & \multicolumn{4}{|c|}{ ppm } & \multicolumn{3}{|c|}{ méq/100g } & & & $\%$ \\
\hline R1V6 & $0-20$ & 7,65 & 30 & 0,13 & 0,01 & 12,15 & 9,21 & 6,47 & 3,2 & 2,2 & 0,02 & 0,02 & 5,44 & 7,75 & 0,70 \\
\hline R1V5 & $0-20$ & 7,45 & 20 & 0,10 & 0,01 & 12,45 & 8,50 & 5,76 & 3,6 & 2,1 & 0,02 & 0,01 & 5,73 & 7,75 & 0,74 \\
\hline R2V6 & $0-20$ & 8,9 & 50 & 0,10 & 0,01 & 12,59 & 8,66 & 5,92 & 3,6 & 1,2 & 0,02 & 0,02 & 4,84 & 7,5 & 0,65 \\
\hline R2V8 & $0-20$ & 9,8 & 60 & 0,10 & 0,01 & 12,59 & 8,55 & 5,81 & 3 & 1,4 & 0,02 & 0,01 & 4,43 & 7,5 & 0,59 \\
\hline
\end{tabular}

\section{DISCUSSION}

Selon les observations faites sur les profils (PGK1 et PGK2) et les sondages à la tarière de la partie cultivées du site de Guillé koira, on peut dire que les horizons de surfaces du sol de ce site sont pauvres en argile avec une forte teneur en éléments grossiers, sa couleur est jaune rougeâtre à sec. L'analyse granulométrique de ce sol nous a permis de constater que la texture sablolimoneuse est dominante. Ce sol sablolimoneux a une bonne porosité et une capacité de rétention en eau faible. Le $\mathrm{pH}$ de ce sol est acide sur les premiers horizons. Cette acidité $(5,05)$ dépasse 5,5 valeur en dessous de laquelle la présence de l'aluminium échangeable présente des risques de toxicités aluminiques (Ambouta, 2015), elle est donc préjudiciable pour le riz. Les taux en carbone, en azote et en phosphore de ce sol sont faibles. La somme des bases échangeables est bonne (le complexe absorbant est riche en $\mathrm{Ca}^{++}$et $\mathrm{Mg}^{++}$mais pauvre en $\mathrm{k}^{+}$). Le rapport $\mathrm{C} / \mathrm{N}$ est bas.

Concernant le site de Lassourou, les observations sur le profil (PLAS 1) et les sondages montrent que les horizons de surfaces sont aussi pauvres en élément fins mais riche en éléments grossiers. De l'analyse granulométrique de ce sol, on déduit que la texture sableuse domine l'horizon de surface. L'analyse chimique a révélé un $\mathrm{pH}$ basique dans les horizons de surface. Ce $\mathrm{pH}$ élevé serait lié à la présence de la nappe phréatique salée à seulement $60 \mathrm{~cm}$. Dans les régions arides et semi-arides, les sols «salins» correspondent normalement aux sols à $\mathrm{pH}$ inférieurs à 8,5 et ont une conductivité électrique de l'extrait de saturation supérieur à $4 \mathrm{mmho} / \mathrm{cm}$ à $25^{\circ} \mathrm{C}$ (Chaudhary Nanda, 2003). Le riz peut accepter des sols à $\mathrm{pH}$ variant entre 4,5 et 8 avec un optimum de l'ordre de 6,5 (ADRAO, 2001). Pour augmenter la production du riz sur ce sol, il faut maintenir en permanence les plants dans l'eau jusqu'au stade laiteux afin de diminuer l'effet de la salinité. Le lessivage et le drainage sont des clés de leur mise en valeur (Arraudeau, M.A., 1998). La Conductivité électrique (CE) $(75)$ et le $\mathrm{pH}(9,2)$ d'une part et la prédominance de la texture grossière en surface d'autre part, occasionne la baisse de rendement de riz. La texture grossière ou sableuse n'est pas très favorable à la riziculture (Angladette, 1976). Elle présente un taux très faible en carbone, en azote et en phosphore. Le rapport $\mathrm{C} / \mathrm{N}$ est bas. La somme des bases échangeables est bonne (le complexe absorbant est aussi riche en $\mathrm{Ca}^{++}$et $\mathrm{Mg}^{++}$mais pauvre en $\mathrm{k}^{+}$). 


\section{Conclusion}

A l'issue de cette étude, on peut retenir que les sols de Guillé koira et Lassourou ont des caractéristiques physicochimiques différentes. Ces sols sont aptes à la riziculture autour des mares mais avec des amendements notamment à Lassourou ou la salinité, le $\mathrm{pH}$ alcalins et la texture grossière ou sableuse sont des facteurs préjudiciables à la riziculture. A cet effet, un apport d'eau régulier en période de sécheresse, un labour profond, l'utilisation rationnelle de la fumure organique et du gypse en fonction des propriétés de sol, la culture des variétés tolérantes à la salinité permettraient d'augmenter significativement la production du riz au niveau de ce site.

L'étude a enfin montré que la composition chimique du sol en particulier le pH et la CE, sont des éléments importants de prise de décision pour la pratique de la riziculture autour des mares.

\section{COMPETING INTERESTS}

Les auteurs déclarent qu'il n'y a aucun conflit d'intérêts pour cet article.

\section{CONTRIBUTIONS DES AUTEURS}

ASY a participé à l'élaboration du protocole expérimental de l'étude, le traitement des données et l'interprétation des résultats ainsi que à la rédaction de cet article. GY a également participé à l'élaboration du protocole expérimental de l'étude et à la rédaction de l'article. MMT a contribué à la fourniture des données notamment celles issues des analyses au laboratoire, leurs interprétation et a également participé à la rédaction de cet article. NAI a contribué à la collecte des données, le traitement et l'interprétation de ces données et à la rédaction de cet article.

\section{REMERCIEMENTS}

Nos sincères remerciements vont au Programme de Productivité Agricole en Afrique de l'Ouest (PPAAO)/ Niger pour le financement de cette étude; à l'Institut National de la Recherche Agronomique du Niger (INRAN), aux facultés des sciences et d'agronomie de l'Université Abdou
Moumouni de Niamey, au directeur général de la Boulangerie Cité Caisse M. Souley Maman Sadi, pour le soutien moral et matériel qu'ils ont apporté dans la réalisation de cette étude; à tous les agents de l'ONG SOS Sahel International-Niger pour leur collaboration dans la réalisation de cette étude.

\section{REFERENCES}

ADRAO. 2001. Collaboration ADRAO/SNRA : Potentiel d'une révolution verte dans le domaine de la riziculture en Afrique de l'ouest et du centre. Rapport de la deuxième réunion biennale ADRAO/comité des experts nationaux, 20-21 mars 2000, M’bé, Bouaké, Cote d'Ivoire $\mathrm{N}^{\circ} 2,110 \mathrm{p}$.

ADRAO. 2004. Vers de nouveaux horizons. Rapport annuel. Bouaké Côte d'Ivoire, $61 \mathrm{p}$.

ADRAO. 2008. Tendances rizicoles en Afrique. Cotonou, Bénin: Centre du riz pour l'Afrique (ADRAO), $84 \mathrm{p}$.

AGDL. 2010. Rapport, 5p.

Ambouta KJM. 2015. Cours de couverture pédologique, Master 2 GISE, faculté d'agronomie de l'UAM de Niamey, 238 diapositives.

Aminatou AB. 2011. Test d'introduction et d'adaptation des variétés de riz pluvial dans la région de Dosso (Boboye et Gaya). Mémoire de fin d'études pour l'obtention du Diplôme d'Etudes Supérieures Spécialisées (DESS), 43p.

Amir S, Saidou AK, Aboubacar I, Moussa S. 2018. Recommendation of a New Fertilizer Rate for Rice in the Irrigated Areas of the Niger River Valley. In Improving the Profitability, Sustainability and Efficiency of Nutrients Through Site Specific Fertilizer Recommendations in West Africa AgroEcosystems (vol 2), Bationo A, Ngaradoum D, Youl S, Lompo F, Fening J (eds). Springer; 37-47. https://doi.org/10.1007/978-3-31958792-9_3

Angladette A. 1976. Le Riz, G-P, (édition Maisonneuve et Larose), Paris, 930 p. 
Arraudeau MA. 1998. Le Riz Irrigué (Tome 1 et Tome II). Édition Maisonneuve et Larose : Paris, France ; 659 p.

Chaudhary N. 2003. Guide d'identification des contraintes de terrain à la production de riz.

FAO. http://www.fao.org/docrep/006/y2778f/y 2778f08.htm\#bm8

FAO. 2004 : Le riz c'est la vie. Billetin trimestriel de statistiques. 23 ème conférence régionale de l'Afrique, année internationale du riz, 1-5 mars 2004, Johannesburg, $129 \mathrm{p}$.

FAO. 2011. Etats des lieux de la riziculture au Niger, 73p. Institut National des Statistique (INS). 2011. Enquête sur la filière rizicole.

Le Houerou HN. 1989. The grazing land ecosystèms of the African Sahel. In Ecological Studies $n^{\circ} 75$. Springes : Berlin, Heidelberg ; 282 p.
PAFRIZ. 2005. Etude sur les modes de faire valoir et le problème sur les Aménagements Hydro Agricoles de la vallée du fleuve Niger.

Sido Yacouba A, Yadji G, Tchicama MM, Issa NA. 2018. Évaluation participative des variétés de riz en riziculture au tour des mares au Niger : cas des communes rurales d'Imanan et de Tondikandia. Journal of Applied Biosciences, 127: 12857-12866.

DOI: https://dx.doi.org/10.4314/jab.v127i1.10

Sido Yacouba A, Zakari Moussa O, Kadri A, Kaka S, Ibroh G. 2013. Participatory Varietal Selection: an approach used for the selection of lowland NERICA varieties in Niger. International Journal of Biological and Chemical Sciences, 7(6): 2202-2213. DOI : http://dx.doi.org/10.4314/ijbcs.v7i6.3 